\title{
Sustained Attention Deficits in Nonpsychotic Relatives of Schizophrenic Patients: A Recurrence Risk Ratio Analysis
}

\author{
Wei J. Chen, Chin-Hao Chang, Shi K. Liu, Tzung J. Hwang, and Hai-Gwo Hwu, collaborators from the \\ Multidimensional Psychopathology Group Research Project
}

\begin{abstract}
Background: In nonpsychotic parents and siblings of schizophrenic patients, the recurrence risk ratios of sustained attention deficits, as measured by the continuous performance test (CPT), were examined with a series of cut-off points.

Metbods: Among 116 parents and 95 siblings of 91 schizophrenic probands in northern Taiwan, both undegraded and degraded sessions of the CPT were administered. Subjects' signal detection sensitivity of CPT performance ( $d^{\prime}$ ) was standardized against a community sample without (unadjusted z score) or with (adjusted z score) adjustment for age, gender, and educational level.

Results: Differences in the risk ratios between the parents and siblings that were based on the unadjusted $\mathrm{z}$ scores of CPT $d^{\prime \prime}$ diminished markedly if the adjusted $\mathrm{z}$ scores were used. As the cut-off point in the adjusted $\mathrm{z}$ score decreased from -2.5 to -3.0 , the risk ratio increased continually for both the undegraded (10.1-18.8 for parents, 10.0-16.7 for siblings) and degraded (12.4-102.7for parents, 8.6-72.0 for siblings) test.
\end{abstract}

Conclusions: Stringent cut-off criteria of CPT deficits with adjustment for demographic features leads to recurrence risk ratios greater than those based on schizophrenia alone in both parents and siblings of schizophrenic patients.

Key Words: Schizophrenia, continuous performance test, sustained attention, recurrence risk ratio, endophenotype

$\mathrm{S}$ ustained attention deficits in schizophrenia, as measured by the continuous performance test (CPT) (Rosvold et al 1956), have become an increasingly important focus in the search for potential endophenotypes for genetic susceptibility to schizophrenia (Chen and Faraone 2000; Cornblatt and Keilp 1994; Cornblatt and Malhotra 2001). Several lines of evidence have converged to support this assertion. For example, deficits on the CPT are present in schizophrenic patients over a range of illness stages (Asarnow and MacCrimmon 1981; Nuechterlein and Dawson 1984; Orzack and Kornetsky 1966), and these are particularly associated with negative and disorganized symptoms (Buchanan et al 1997; Ito et al 1997; Liu et al 1997; Nuechterlein et al 1986; Roitman et al 1997). Furthermore, deficits on more difficult test versions have been found to be more significant (Seidman et al 1998) and not reversed by treatment with neuroleptics (Addington and Addington 1997; Cornblatt et al 1997; Epstein et al 1996; Finkelstein et al 1997; Liu et al 2000). Although patients with major affective disorders might also display such deficits during the acute stage of their illness, they usually improve to normal or a much less affected status when clinical symptoms disappear (Liu et al 2002; Mojtabai et al 2000; Nelson et al 1998). In parallel, nonpsychotic relatives of schizophrenic patients (Chen et al 1998b; Grove et al 1991; Mirsky et al 1995) or people with schizotypal features (Chen et al 1997; Lenzenweger et al 1991; Obiols et al 1993) also exhibit deficits on more difficult versions of the CPT, and the deficits are specifically associated with the negative and disorganization factors of schizotypy.

In the assessment of the magnitude of familial aggregation of a disease or physiologic trait, the recurrence risk ratio $(\lambda)$ is a

From the Institute of Epidemiology (WJC, C-HC, H-GH), College of Public Health, and Department of Psychiatry (WJC, SKL, H-GH), College of Medicine, National Taiwan University; and the Department of Psychiatry (WJC, SKL, TJH, H-GH), National Taiwan University Hospital, Taipei, Taiwan.

Address reprint requests to Wei J. Chen, M.D., National Taiwan University, College of Public Health, Institute of Epidemiology, 1 Jen-Ai Road, Sec. 1, Taipei 100, Taiwan.

Received August 11, 2003; revised January 7, 2004; accepted January 9, 2004. commonly used index (Risch 1990a). In a multilocus multiplicative model, $\lambda$ is found to be proportional to the power of an affected-relative-pair genetic linkage analysis (Risch 1990b). For sustained attention deficit to be a useful endophenotype, the magnitude of its $\lambda$ should be greater than that of the schizophrenia diagnosis itself. Previously, we (Chen et al 1998b) reported in a preliminary sample of parents and siblings of schizophrenic patients that the $\lambda s$ for the CPT deficits might reach as much as 15-30, consistent with the predictions by Faraone et al (1995). Recently, Egan et al (2000) replicated the finding that the risk of attention deficits among nonpsychotic siblings of schizophrenic patients was elevated, although the magnitude of the risk ratio was only $3-5$, not greater than that of schizophrenia itself. The two studies differed in several aspects of methodology, such as sources of schizophrenic probands, types of relatives included, and the sampling and size of the reference sample. A further issue is whether pooling both parents and siblings together would bias the estimate.

In this study, we used an expanded sample from our previous study to estimate the recurrence risk ratio of the CPT deficits at various cut-off points. Issues addressed included 1) whether the $\lambda$ of parents differs from that of siblings; 2 ) the impact of the control for demographic features on the estimates of $\lambda$; and 3 ) the impact of different CPT versions on the estimate of $\lambda$.

\section{Methods and Materials}

\section{Subjects}

Schizophrenic probands were drawn from a 5-year, prospective, clinical study of schizophrenia in Northern Taiwan, the Multidimensional Psychopathology Group Research Project (Chang et al 2002; Chen et al 1998b). Briefly, from August 1, 1993 to June 30, 1998, patients admitted to the acute inpatient psychiatric wards of National Taiwan University Hospital, Taipei City Psychiatric Center, and Taoyuan Psychiatric Center were included. Patients had to fulfill DSM-IV criteria for schizophrenia and the requirements of sampling design, in which the ratio of patients admitted for the first time to those admitted for relapse was set at 1:2 to ensure inclusion of patients in different phases of the illness. Patients with a discharge diagnosis other than schizophrenia and patients with a history of physical illness or substance abuse that made the diagnosis questionable were excluded. 
Eligible schizophrenic patients were further included as the probands of the present family genetic study when they fulfilled the following inclusion criteria: 1) the proband agreed to let researchers meet with their relatives; 2) at least one of their first-degree relatives agreed to participate; and 3) more than one half of their first-degree relatives were logistically accessible (living within Northern Taiwan, from Hsinchu County to Yilan County). All the first-degree relatives aged 16 years or older were included in the present study. Written informed consent was obtained from all subjects after complete description of the study. The study was approved by the institutional review boards of the participating hospitals.

Among 234 schizophrenic patients recruited, 94 (40.2\%) met the criteria to be included for this family study (Chang et al 2002). When the participating and nonparticipating probands were compared, there was no difference in ethnicity; however, the nonparticipating probands were older $(32.2 \pm 7.1$ years vs. 28.9 \pm 7.3 years) and had a lower education level ( $10.5 \pm 2.8$ years vs. $11.4 \pm 3.0$ years) than the participating ones. Among a total of 450 first-degree relatives, 441 were aged 16 years or older. Of these, 45 were excluded from interviewing (30 were dead, 13 were abroad, and two were profoundly mentally retarded), and 234 (59.1\%) of the remaining 396 were directly interviewed. The completion rate was higher in parents $(80 \%)$ than in siblings (42\%). Three schizophrenic probands who did not complete at least one session of the CPT were deleted from subsequent analyses. Among the relatives, six had a diagnosis of schizophrenia, one schizophreniform disorder, two mental retardation, 10 alcohol abuse or dependence, and one drug abuse; these were excluded from subsequent analyses. In total, 91 schizophrenic probands and 211 first-degree relatives were included for the analyses. Compared with the first-degree relatives who were included in this study, those not included had a higher probability of being male, young in age, and sibling but were not different in educational level.

To provide estimates of the population prevalence of the sustained attention deficits at various cut-off points, a representative sample of community subjects was recruited (Chen et al 1998a). Briefly, adults aged 20-65 years were systematically sampled from the 1993 and 1994 voter lists of Chinshan Township, north of Taipei. Among a total of 880 selected adults, 215 did not live in Chinshan, and 102 could not be located. For the remaining 563 subjects who could be contacted, 365 (64.8\%) completed a questionnaire on schizotypal personality after informed written consent had been obtained. The reasons for noncompletion included the following: 120 (60.9\%) failed to show up for appointment, 50 (25.4\%) refused to take the test, 23 (11.7\%) were in military service, four (2.0\%) were overseas fishing, and one remained silent during interview. Among the 365 adult subjects who completed the questionnaire, 18 did not complete the CPT testing, and two were excluded because of a diagnosis of either schizophrenia or stroke. Overall, 345 adult subjects who did not have a diagnosis of psychosis or stroke and completed both the undegraded and the degraded CPT were included as the reference sample of the test.

\section{Interview Instruments and Diagnostic Procedure}

Schizophrenic probands and their first-degree relatives were interviewed in person with the Diagnostic Interview for Genetic Studies (Nurnberger et al 1994). The establishment of the Chinese version and its interrater reliability have been described in our earlier report (Chen et al 1998b). For those relatives who were not directly interviewed, interviewers used the Chinese version of the Family Interview for Genetic Studies (NIMH Genetics Initiative 1992) to collect relevant information from other family members.

Two psychiatrists and one psychiatric epidemiologist independently assessed the diagnosis of each subject by reviewing the subject's results on the Diagnostic Interview for Genetic Studies and other available information, including hospital records and the interviewer's notes. Best-estimate lifetime psychiatric diagnoses according to the DSM-IV criteria were determined independently and then finalized in a consensus meeting.

\section{Continuous Performance Test}

The participants were assessed with the CPT, version 2.20, operated on a Sunrise Systems machine (Pembroke, Massachusetts). As described by Chen et al (1998a), numbers from 0-9 were randomly presented for $50 \mathrm{msec}$ each, at a rate of one per second. Each subject undertook two CPT sessions: the undegraded 1-9 task and the degraded 1-9 task, in which subjects were asked to respond whenever the number "9" preceded by the number " 1 " appeared on the screen. A total of 331 trials, 34 (10\%) of them targets, were presented over $5 \mathrm{~min}$ for each session. During the $25 \%$ degraded session, a pattern of snow was used to toggle background and foreground dots so that the image was not distinct.

Raw scores on the CPT performance were expressed in two signal-detection indices on the test—sensitivity $\left(\mathrm{d}^{\prime}\right)$ and response criterion (natural logarithm of $\beta[\ln \beta]$ )—which were derived from the hit rate (probability of response to target trials) and false-alarm rate (probability of response to nontarget trials) under the assumption of the normal distribution and equal variances of signal and noise (Nuechterlein 1991). Sensitivity measures an individual's ability to discriminate target stimuli from nontarget stimuli, whereas response criterion measures the amount of perceptual evidence that the person requires to identify a stimulus as a target. Variables extraneous to the intended comparison of sensitivity, such as differences between groups in level of motivation or cooperativeness, would yield differences in $\ln \beta$ rather than in $\mathrm{d}^{\prime}$. In a 1 -week test-retest reliability study of the CPT versions used in this study, the intraclass correlation coefficients of reliability of $\mathrm{d}^{\prime}$ were .83 and .82 for the undegraded and the $25 \%$ degraded task, respectively, and those of $\ln \beta$ were .49 and .72 for the undegraded and the 25\% degraded task, respectively (Chen et al 1998a).

\section{Statistical Analysis}

Each subject's CPT performance raw scores were 1) merely standardized by use of the mean and SD of the community subjects, expressed as unadjusted $z$ score; and 2) standardized with adjustments for gender, age, and education, as described by Chen et al (1998b), expressed as adjusted $z$ score. Briefly, the predictive score of a subject was calculated by use of the regression coefficients obtained from the regression of the scores on these covariates among the 345 community subjects. The difference between the raw score and the predictive score was then standardized by the root mean error of the regression and was defined as the adjusted $z$ score of the subject. For comparison between two groups, Fisher's exact test (two-tailed) was used for dichotomous variables, and $t$ test (two-tailed) was used for continuous variables.

Subjects were designated as affected with attention deficit if their $z$ scores of the CPT $\mathrm{d}^{\prime}$ fell below a prespecified cut-off value. A series of cut-off points was chosen: 1, 2, 2.5, 2.75, and 3 SDs below the population mean. The $\lambda$ was calculated as the 
Table 1. Demographic Features and Continuous Performance Test Scores for Study Subjects

\begin{tabular}{|c|c|c|c|c|}
\hline Variable & $\begin{array}{l}\text { Schizophrenic Probands } \\
\qquad(n=91)^{a}\end{array}$ & $\begin{array}{l}\text { Parents } \\
(n=116)^{b}\end{array}$ & $\begin{array}{l}\text { Siblings } \\
(n=95)^{c}\end{array}$ & $\begin{array}{c}\text { Community Sample } \\
(n=345)\end{array}$ \\
\hline Male, $n(\%)$ & $49(54)$ & $46(40)$ & $47(49)$ & $165(48)$ \\
\hline Age (years), Mean (SD) & $28.8(7.4)$ & $56.8(9.1)$ & $32.3(10.1)$ & $41.3(13.0)$ \\
\hline Education (years), Mean (SD) & $11.3(3.0)$ & $7.4(4.8)$ & $12.3(3.0)$ & $7.4(4.3)$ \\
\hline \multicolumn{5}{|l|}{ Raw Score on the Test, Mean (SD) } \\
\hline $\mathrm{d}^{\prime}$ on the undegraded test & $2.3(1.8)$ & $3.1(1.6)$ & $3.7(1.2)$ & $4.0(.8)$ \\
\hline$d^{\prime}$ on the $25 \%$ degraded test & $1.4(1.7)$ & $2.2(1.7)$ & $3.0(1.7)$ & $3.3(1.0)$ \\
\hline $\ln \beta$ on the undegraded test & $1.6(1.8)$ & $1.9(1.6)$ & $2.2(1.0)$ & $2.2(.9)$ \\
\hline $\ln \beta$ on the $25 \%$ degraded test & $1.2(1.8)$ & $1.4(1.8)$ & $1.9(1.4)$ & $2.2(.9)$ \\
\hline \multicolumn{5}{|c|}{ Adjusted $z$ Score on the Test, Mean (SD) } \\
\hline $\mathrm{d}^{\prime}$ on the undegraded test & $-3.0(2.5)$ & $-.9(2.1)$ & $-1.0(1.6)$ & $0(1.0)$ \\
\hline$d^{\prime}$ on the $25 \%$ degraded test & $-2.6(1.8)$ & $-.9(1.9)$ & $-.8(1.7)$ & $0(1.0)$ \\
\hline In $\beta$ on the undegraded test & $-.6(2.1)$ & $-.5(1.9)$ & $.1(1.2)$ & $0(1.0)$ \\
\hline $\ln \beta$ on the $25 \%$ degraded test & $-1.1(1.9)$ & $-.9(2.0)$ & $-.2(1.5)$ & $0(1.0)$ \\
\hline
\end{tabular}

${ }^{a}$ Five missing for the degraded test.

${ }^{b}$ Six missing for the degraded test.

'Two missing for the degraded test.

ratio of the recurrence risk among a certain type of relatives to the prevalence of the general population.

For estimation of recurrence risk, only the relatives of those probands whose CPT scores fell below a specified cut-off value were included for the estimation. Two siblings who had schizophrenia were not included for the analyses here because they did not take the CPT. Families included for the analyses at a series of cut-off points can thus be treated as ascertained under single ascertainment. A recent study has shown that the unweighted estimator of the recurrence risk is a consistent estimator of sibling recurrence risk under single ascertainment (Olson and Cordell 2000). The recurrence risk was hence calculated as the number of affected relatives divided by all nonpsychotic relatives pooled together. The prevalence of the deficit on the CPT in the general population was estimated from the 354 community subjects with the corresponding cut-off values in the $z$ scores of CPT d'.

\section{Results}

The mean age of schizophrenic probands was 28.8 years, slightly younger than their siblings, whereas the distribution of gender and educational level were similar between the schizophrenic probands and their siblings (Table 1). The parents of the schizophrenic probands had lower educational levels than their offspring, which was mainly owing to the limited educational resources for the parents' generation while it came of age in Taiwan. For the mean raw scores of $\mathrm{d}^{\prime}$ on both versions of the test, the schizophrenic probands had the lowest value, followed by the parents and then the siblings, as compared with the community sample (all $p$ values of the $t$ test $<.05$ ). In contrast, the mean raw scores of $\ln \beta$ for the siblings were not different from those of the community sample. The raw scores of $\ln \beta$ for the schizophrenic probands and their parents were only slightly less than those of the community sample. As expected, the mean adjusted $z$ scores of $\mathrm{d}^{\prime}$ for the schizophrenic probands and their parents and siblings were less than 0 , with the probands having the lowest values, followed by the parents and the siblings. In contrast, the mean adjusted $z$ scores of $\ln \beta$ for the siblings were near 0 , and those of the schizophrenic probands and their parents were at most 1 SD less than zero.

When the criterion of attention deficit as defined by the unadjusted $z$ score of $\mathrm{d}^{\prime}$ became more stringent, the proportion of the deficit in both the parents and the siblings decreased substantially for the lowering of the cut-off point from -1 to -2 and then remained relatively steady for the lowering from -2 to -3 (Table 2). This indicates that there was indeed a core subgroup of relatives who were markedly deviant on the CPT. Meanwhile, the prevalence of the deficit in the community sample decreased even more sharply. Thus, the $\lambda$ in both the parents and the siblings increased as the threshold became more stringent. At cut-off points from -1.0 to -2.0 , the $\lambda s$ of the degraded test were slightly greater than those of the undegraded test. When the cut-off point moved to -2.75 or -3.0 , however, the $\lambda s$ of the undegraded test became much greater than those of the degraded test. The parents had greater $\lambda s$ than the siblings across all cut-off points.

The proportions of the deficit based on the adjusted $z$ scores are displayed in Table 3 . The $\lambda s$ of the degraded test were greater than those of the undegraded test at all cut-off points in the parents and at the majority of the cut-off points in the siblings. When the two types of relatives were compared, the $\lambda s$ of the undegraded test for the parents were either slightly less or slightly greater than those for the siblings, whereas the $\lambda s$ of the degraded test in the parents were consistently greater across all cut-off points than those in the siblings.

The impact of controlling for the demographic features on the estimates of risk ratios could be evaluated by comparing the values in Table 2 with those in Table 3, as displayed in Figure 1. First, for cut-off points $\geq-2.5$, the difference between the $\lambda s$ based on the unadjusted $z$ scores and those based on the adjusted $z$ scores were relatively small. When the cut-off points were more stringent than -2.5 , the magnitude of $\lambda$ s based on the adjusted $z$ score became much less than those based on the unadjusted $z$ scores for the undegraded test, whereas the magnitude of $\lambda s$ based on the adjusted $z$ score became much greater than those based on the unadjusted $z$ scores for the degraded test. Second, the $\lambda s$ of the undegraded test were no longer greater than those of the degraded test when the adjusted $z$ scores were used. Instead, the $\lambda s$ of the degraded test were greater than those of the undegraded test for both the parents and the siblings. Third, the differences between the parents and the siblings in the $\lambda s$ became less $(<3)$ when the adjusted $z$ scores were used except for the degraded test at the cut-off points of -2.75 and -3 .

\section{Discussion}

Ascertainment bias is a common problem encountered in the estimation of $\lambda$ because the relatives are identified through 
Table 2. Proportion of Subjects Whose Unadjusted $z$ Score of $d^{\prime}$ on the Continuous Performance Test Was Below a Threshold and the Corresponding Recurrence Risk Ratio at Various Cut-Off Points

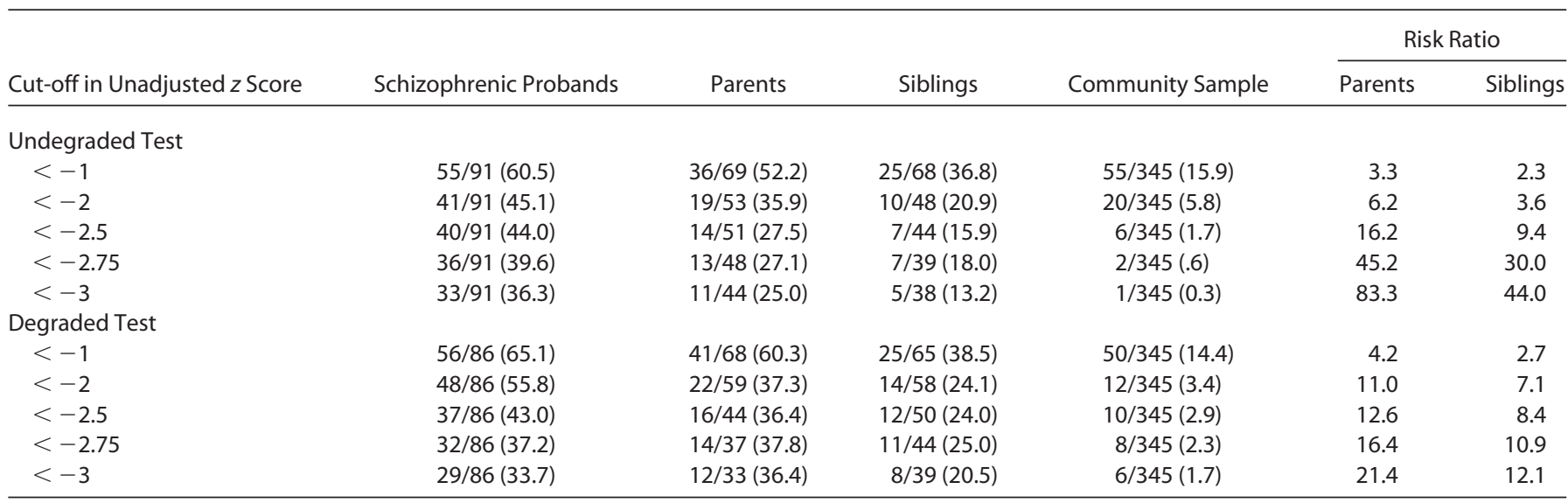

Data other than risk ratio are presented as $n / N(\%)$. The denominator of relatives would decrease as the threshold became more stringent, whereas the denominator of probands and normal comparison subjects remained the same across all cut-off points.

affected probands (Guo 1998). In this study, the analyses were limited to the relatives whose schizophrenic probands also had deficits on the CPT. Thus, it is reasonable to assume single ascertainment, and the estimator used in this study is corrected for ascertainment bias (Olson and Cordell 2000); however, the participation rate among the eligible siblings (42\%) was a concern, because it was much lower than that among the eligible parents (80\%). This might reflect the strong social stigma against schizophrenia in Taiwanese society, which might prevent siblings from exposing themselves to inquiry about the illness. In addition, some siblings might decline participation because of their schizotypal personality features (Norden et al 1995), which have been found to be associated with poorer sustained attention (Chen et al 1998b). Hence, the proportion of siblings with sustained attention deficit in this study might be underestimated and the $\lambda$ underestimated as well.

An important assumption of the current risk ratio analysis is that a person's CPT performance is stable. The risk ratio estimates might vary if a person's test performance changes with time, as revealed in a recent longitudinal analysis on cardiovascular risk factors (Chen et al 2003). Our classification of probands' attention status was based on their CPT administered shortly after their admission to psychiatric wards. Although previous studies have indicated that schizophrenic patients' CPT performance deficits on more difficult versions were stable at least in a period up to 3 months (Addington and Addington 1997; Cornblatt et al 1997; Epstein et al 1996; Finkelstein et al 1997; Liu et al 2000), whether this is true for a longer period remains to be investigated.

One concern over the performance deficits on the CPT is subjects' lack of motivation or cooperation. Indeed, the mean response criterion adopted by the schizophrenic probands and their parents was less than that of the community sample, though not less than 1 SD. In contrast, the siblings adopted a response criterion similar to that of the community sample; however, the impact of this in parents seemed minimal because the mean adjusted $z$ scores on both the undegraded and degraded CPT for the parents were almost similar to those for the siblings.

Although some of the $\lambda$ estimates were similar between those based on the unadjusted $z$ scores of $\mathrm{d}^{\prime}$ and those based on the adjusted $z$ scores of $\mathrm{d}^{\prime}$, many were considerably different. The differences in the $\lambda$ estimates between the parents and the siblings diminished markedly after the demographic features were controlled for. Some changes in $\lambda$ estimates were so substantial that they reversed the overall trend. For example, the estimates of $\lambda$ based on the unadjusted $z$ score of the undegraded test were much greater than those of the degraded test at cut-off

Table 3. Proportion of Subjects Whose Adjusted $z$ Score of $d^{\prime}$ on the Continuous Performance Test Was Below a Threshold and the Corresponding Recurrence Risk Ratio at Various Cut-Off Points

\begin{tabular}{|c|c|c|c|c|c|c|}
\hline Cut-Off in Adjusted $z$ Score & Schizophrenic Probands & Parents & Siblings & Community Sample & \multicolumn{2}{|c|}{ Risk Ratio } \\
\hline Undegraded Test & & & & & & \\
\hline$<-2$ & $51 / 91(56.1)$ & $21 / 66(31.8)$ & $23 / 64(35.9)$ & $15 / 345(4.4)$ & 7.2 & 8.2 \\
\hline$<-2.5$ & $45 / 91(49.5)$ & $15 / 57(26.3)$ & $14 / 54(25.9)$ & $9 / 345(2.6)$ & 10.1 & 10.0 \\
\hline$<-2.75$ & $42 / 91(46.2)$ & $12 / 54(22.2)$ & $11 / 49(22.5)$ & $6 / 345(1.8)$ & 12.3 & 12.5 \\
\hline$<-1$ & $63 / 86(73.3)$ & $45 / 77(58.4)$ & $36 / 72(50.0)$ & $48 / 345(13.9)$ & 4.2 & 3.6 \\
\hline$<-2$ & $53 / 86(61.7)$ & $23 / 66(34.9)$ & $19 / 61(31.2)$ & $16 / 345(4.6)$ & 7.6 & 6.8 \\
\hline$<-2.5$ & $50 / 86(58.2)$ & $20 / 62(32.3)$ & $13 / 58(22.4)$ & $9 / 345(2.6)$ & 12.4 & 8.6 \\
\hline$<-2.75$ & $44 / 86(51.2)$ & $16 / 53(30.2)$ & $12 / 54(22.2)$ & $5 / 345(1.5)$ & 20.1 & 14.8 \\
\hline$<-3$ & $41 / 86(47.7)$ & $16 / 52(30.8)$ & $11 / 51(21.6)$ & $1 / 345(.3)$ & 102.7 & 72.0 \\
\hline
\end{tabular}

Data other than risk ratio are presented as $n / N(\%)$. The denominator of relatives would decrease as the threshold became more stringent, whereas the denominator of probands and normal comparison subjects remained the same across all cut-off points. 
A

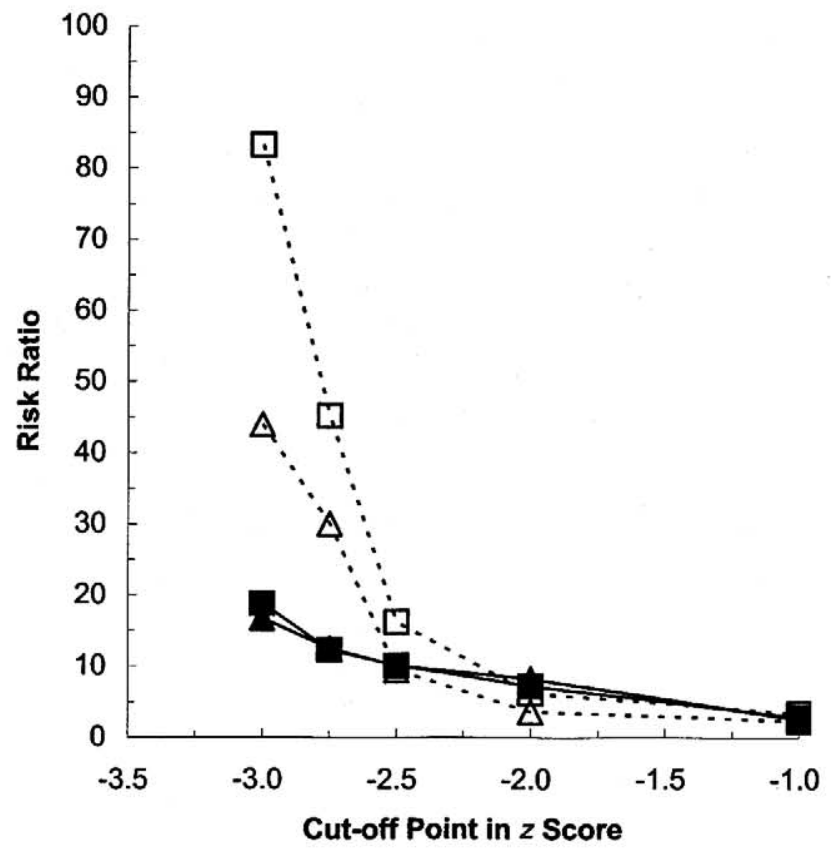

B

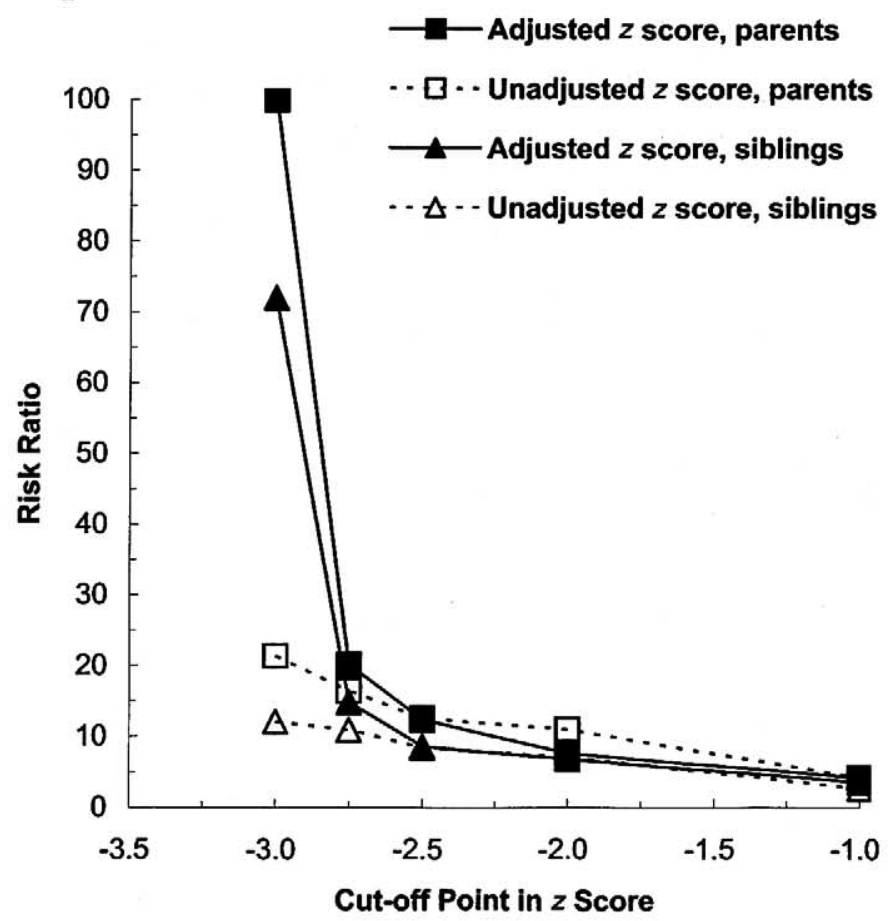

Figure 1. Recurrence risk ratio $(\lambda)$ at various cut-off points in $z$ score on the (A) undegraded and the (B) degraded continuous performance test for the nonpsychotic parents $(n=116)$ and siblings $(n=95)$ of schizophrenic patients $(n=91)$, as standardized against a community sample $(n=345)$ without (unadjusted $z$ score) or with (adjusted $z$ score) adjustment for age, gender, and educational level.

points more stringent than -2 ; however, similar estimates of $\lambda$ based on the adjusted $z$ score of the undegraded test turned out to be less than those of the degraded test. These findings reinforce the notion that a sufficient size of the community sample is essential for comparison of CPT scores across groups of subjects.

Another concern related to our earlier report, raised by Egan et al (2000), was that the $\lambda s$ might be overestimated because parents and siblings were pooled together for the estimation. In the present study, the $\lambda s$ based on the unadjusted $z$ scores among the parents were indeed greater than those among the siblings; however, this was most likely due to the older age and lower educational level among the parents as compared with the siblings. When the adjusted $z$ scores were used for the estimation, the values of the two groups became very close to each other, though parents' $\lambda$ was still slightly greater than siblings'.

The CPT used in this study includes a working memory component in both versions and additional perceptual load in the degraded one (Nuechterlein et al 1992). The $\lambda$ s based on the degraded test were found to be greater than those based on the undegraded test after the demographic features were controlled for. The difference between the two estimates became more apparent when more stringent criteria were chosen. These results indicate that a more difficult test and/or a more stringent criterion lead to a higher risk ratio. Particularly, the $\lambda$ for the cut-off point at -2.5 or -3 is greater than the $\lambda$ that is based on the clinical phenotype of schizophrenia (approximately 10). This is consistent with previous studies (Chen and Faraone 2000; Liu et al 2002) in supporting the proposition that the different CPT versions might tap into different components of sustained attention: the more difficult versions are stable vulnerability indicators, whereas the simpler versions might be more state-depen- dent. With a cut-off point of -3 , approximately $44 \%-48 \%$ of schizophrenic probands were below the threshold, which might be a homogeneous subgroup for genetic dissection; however, very few community subjects whose degraded CPT scores were less than -3 and render the $\lambda$ estimates unstable.

Intriguingly, Egan et al (2000) found that the risk ratios at corresponding cut-off points in siblings of schizophrenic probands were only approximately $2-5$. Several reasons might account for this. First, the probands recruited by Egan et al, who were outpatients and had a mean d' less than 1 SD below that of the control group, had less impairment in the sustained attention as compared with the probands in this study. Second, the size of the sample used for the estimation of population prevalence was much smaller $(n=45)$ in the Egan et al study as compared with our community sample $(n=345)$, which might affect the estimation of SD for the scores on the test. Our results indicate that the estimation of $\lambda$ was sensitive to the number of the community sample exceeding a stringent criterion. Third, the CPT versions used by Egan et al seem to be less difficult than ours, because their controls had a higher mean raw score of $\mathrm{d}^{\prime}$ (ranging from 4.80 to 4.70) and lower mean $\ln \beta$ (ranging from .30 to .37 ) than the community sample in this study.

Although $\lambda$ indicates the overall relative risk, it does not necessarily reflect the effect for a specific gene. For example, a recent study has revealed that $\lambda$ is influenced by the allele frequency and mode of inheritance of the trait locus (Rybicki and Elston 2000). Thus, by itself $\lambda$ does not provide a reliable parameter for estimating the statistical power of a proposed linkage study. Nevertheless, an accurate estimate of $\lambda$ can still serve as a prelaboratory assessment of the genetic effect conferred to the trait under study. An alternative approach toward gene mapping for the sustained attention in the families of 
schizophrenic patients is to treat the scores on the CPT as continuous and use the methods for quantitative-trait locus mapping. This might boost the statistical power further for linkage analysis (Risch 2000).

In conclusion, sustained attention deficits, defined by being more than 2.5 SDs below the population mean, are present in approximately half of schizophrenic patients and indicate higher recurrence risk ratios than those based on schizophrenia alone among their parents and siblings as well.

Other participants in the Multidimensional Psychopathology Group Research Projects who helped in the recruitment and evaluation of schizophrenic patients include Drs. Ching-Jui Chang, Hung-Jung Chang, Hai Ho, Ping-Ju Chang, Shi-Chin Guo, Shian-Yuan Lan, Su-Kuan Lin, Fu-Chuan Wei, and Joseph J. Cheng. We thank Miss Shib-Wei Lin for her help in compiling relevant data.

This research was supported by grants from the National Health Research Institutes, Taiwan (DOH 88-HR-825, NHRI-GTEX89P825P, NHRI-EX91-9113PP) and National Science Council, Taiwan (NSC83-0412-B-002-310, NSC84-2331-B-002-187).

Addington J, Addington D (1997): Attentional vulnerability indicators in schizophrenia and bipolar disorder. Schizophr Res 23:197-204.

Asarnow RF, MacCrimmon DJ (1981): Span of apprehension deficits during the postpsychotic stages of schizophrenia: A replication and extension. Arch Gen Psychiatry 38:1006-1011.

Buchanan RW, Strauss ME, Breier A, Kirkpatrick B, Carpenter WT Jr. (1997): Attentional impairments in deficit and nondeficit forms of schizophrenia. Am J Psychiatry 154:363-370.

Chang C-J, Chen WJ, Liu S-K, Cheng JJ, Ou Yang W-C, Chang H-J, et al (2002): Morbidity risk of psychiatric disorders among the first degree relatives of schizophrenia patients in Taiwan. Schizophr Bull 28:379-392.

Chen WJ, Faraone SV (2000): Sustained attention deficits as markers of genetic susceptibility to schizophrenia. Am J Med Genet 97:52-57.

Chen WJ, Hsiao CK, Hsiao L-L, Hwu H-G (1998a): Performance of the Continuous Performance Test among community samples. Schizophr Bull 24:163-174.

Chen WJ, Hsiao CK, Lin CCH (1997): Schizotypy in community samples: The three-factor structure and correlation with sustained attention. J Abnorm Psychol 106:649-654.

Chen WJ, Liu P-H, Ho Y-Y, Chien K-L, Lo M-T, Shih W-L, et al (2003): Sibling recurrence risk ratio analysis of metabolic syndrome and its components over time. BMC Genet 4(Suppl1):S33.

Chen WJ, Liu SK, Chang C-J, Lien Y-J, Chang Y-H, Hwu H-G (1998b): Sustained attention deficit and schizotypal personality features in nonpsychotic relatives of schizophrenic patients. Am J Psychiatry 155:1214-1220.

Cornblatt BA, Keilp JG (1994): Impaired attention, genetics, and the pathophysiology of schizophrenia. Schizophr Bull 20:31-46.

Cornblatt BA, Obuchowski M, Schnur B, O'Brien JD (1997): Attention and clinical symptoms in schizophrenia. Psychiatr Q 68:343-359.

Cornblatt BA, Malhotra AK (2001): Impaired attention as an endophenotype for molecular genetic studies of schizophrenia. Am J Med Genet 105:1115.

Egan MF, Goldberg TE, Gscheidle T, Weirich M, Bigelow LB, Weinberger DR (2000): Relative risk of attention deficits in siblings of patients with schizophrenia. Am J Psychiatry 157:1309-1316.

Epstein Jl, Keefe RSE, Roitman SL, Harvey PD, Mohs RC (1996): Impact of neuroleptic medications on continuous performance test measures in schizophrenia. Biol Psychiatry 39:902-905.

Faraone SV, Kremen WS, Lyons MJ, Pepple JR, Seidman LJ, Tsuang MT (1995): Diagnostic accuracy and linkage analysis: How useful are schizophrenia spectrum phenotypes? Am J Psychiatry 152:1286-1290.

Finkelstein JRJ, Cannon TD, Gur RE, Gur RC, Moberg P (1997): Attentional dysfunctions in neuroleptic-naive and neuroleptic-withdrawn schizophrenic patients and their siblings. J Abnorm Psychol 106:203-212.

Grove WM, Lebow BS, Clementz BA, Cerri A, Medus C, lacono WG (1991): Familial prevalence and coaggregation of schizotypal indicators: A multitrait family study. J Abnorm Psychol 100:115-121.
Guo S-W (1998): Inflation of sibling recurrence-risk ratio, due to ascertainment bias and/or overreporting. Am J Hum Genet 63:252-258.

Ito M, Kanno M, Mori Y, Niwa S (1997): Attention deficits assessed by Continuous Performance Test and Span of Apprehension Test in Japanese schizophrenic patients. Schizophr Res 23:205-211.

Lenzenweger MF, Cornblatt BA, Putnick M (1991): Schizotypy and sustained attention. J Abnorm Psychol 100:84-89.

Liu SK, Chen WJ, Chang C-J, Lin H-N (2000): Effects of atypical neuroleptics on sustained attention deficits in schizophrenia: $A$ trial of risperidone versus haloperidol. Neuropsychopharmacol 22:311-319.

Liu SK, Chiu C-H, Hwang T-J, Chang C-J, Hwu HG, Chen WJ (2002): Deficits in sustained attention in schizophrenia and affective disorders: Stable versus state-dependent markers. Am J Psychiatry 159:975-982.

Liu SK, Hwu H-G, Chen WJ (1997): Clinical symptom dimensions and deficits on the Continuous Performance Test in schizophrenia. Schizophr Res 25:211-219.

Mirsky AF, Yardley SL, Jones BP, Walsh D, Kendler KS (1995): Analysis of the attention deficit in schizophrenia: A study of patients and their relatives in Ireland. J Psychiatr Res 29:23-42.

Mojtabai R, Bromet EJ, Harvey PD, Carlson GA, Craig TJ, Fennig S (2000): Neuropsychological differences between first-admission schizophrenia and psychotic affective disorder. Am J Psychiatry 157:1453-1460.

Nelson EB, Sax KW, Strakowski SM (1998): Attentional performance in patients with psychotic and nonpsychotic major depression and schizophrenia. Am J Psychiatry 155:137-139.

NIMH Genetics Initiative (1992): Family Interview for Genetic Studies. Rockville, Maryland: National Institute of Mental Health.

Norden KA, Klein DN, Ferro T, Kasch K (1995): Who participates in a family study? Compr Psychiatry 36:199-206.

Nuechterlein KH (1991): Vigilance in schizophrenia and related disorders. In: Steinhauer SR, Gruzelier JH, Zubin J, editors. Handbook of Schizophrenia, Vol. 5. Neuropsychology, Psychophysiology and Information Processing. Amsterdam: Elsevier, 397-433.

Nuechterlein KH, Dawson ME (1984): Information processing and attentional functioning in the developmental course of schizophrenic disorders. Schizophr Bull 10:160-203.

Nuechterlein KH, Dawson ME, Gitlin M, Ventura J, Goldstein MJ, Snyder KS, et al (1992): Developmental processes in schizophrenic disorders: Longitudinal studies of vulnerability and stress. Schizophr Bull 18:387-425.

Nuechterlein KH, Edell ES, Norris M, Dawson ME (1986): Attentional vulnerability indicators, thought disorder, and negative symptoms. Schizophr Bull 12:408-426.

Nurnberger JI Jr, Blehar MC, Kaufmann CA, York-Cooler C, Simpson SG, Harkavy-Friedman J, et al (1994): Diagnostic Interview for Genetic Studies: Rationale, unique features, and training. Arch Gen Psychiatry 51:849859.

Obiols JE, García-Domingo M, de Trinchería I, Doménech E (1993): Psychometric schizotypy and sustained attention in young males. Pers Indiv Diff 14:381-384.

Olson JM, Cordell HJ (2000): Ascertainment bias in the estimation of sibling genetic risk parameters. Genet Epidemiol 18:217-235.

Orzack MH, Kornetsky C (1966): Attention dysfunction in chronic schizophrenia. Arch Gen Psychiatry 14:323-327.

Risch N (1990a): Linkage strategies for genetically complex traits, I: Multilocus models. Am J Hum Genet 46:222-228.

Risch N (1990b): Linkage strategies for genetically complex traits, II: The power of affected relative pairs. Am J Hum Genet 46:229-241.

Risch NJ (2000): Searching for genetic determinants in the new millennium. Nature 405:847-856.

Roitman SEL, Keefe RSE, Harvey PD, Siever LJ, Mohs RC (1997): Attentional and eye tracking deficits correlate with negative symptoms in schizophrenia. Schizophr Res 26:139-146.

Rosvold HE, Mirsky AF, Sarason I, Bransome ED Jr, Beck LH (1956): A Continuous Performance Test of brain damage. J Consult Psychol 20:343-350.

Rybicki BA, Elston RC (2000): The relationship between the sibling recurrence-risk ratio and genotype relative risk. Am J Hum Genet 66:593-604.

Seidman LJ, Van Manen K-J, Turner WM, Gamser DM, Faraone SV, Goldstein $J M$, et al (1998): The effects of increasing resource demand on vigilance performance in adults with schizophrenia or developmental attentional/learning disorders: A preliminary study. Schizophr Res 34:101112. 